\title{
Aminoalkyl radicals as halogen-atom transfer agents for activation of alkyl and aryl halides
}

DOI:

10.1126/science.aba2419

\section{Document Version}

Accepted author manuscript

Link to publication record in Manchester Research Explorer

\section{Citation for published version (APA):}

Constantin, T., Zanini, M., Regni, A., Sheikh, N. S., Julia Hernandez, F., \& Leonori, D. (2020). Aminoalkyl radicals as halogen-atom transfer agents for activation of alkyl and aryl halides. Science, 367(6481), 1021-1026.

https://doi.org/10.1126/science.aba2419

\section{Published in:}

Science

\section{Citing this paper}

Please note that where the full-text provided on Manchester Research Explorer is the Author Accepted Manuscript or Proof version this may differ from the final Published version. If citing, it is advised that you check and use the publisher's definitive version.

\section{General rights}

Copyright and moral rights for the publications made accessible in the Research Explorer are retained by the authors and/or other copyright owners and it is a condition of accessing publications that users recognise and abide by the legal requirements associated with these rights.

\section{Takedown policy}

If you believe that this document breaches copyright please refer to the University of Manchester's Takedown Procedures [http://man.ac.uk/04Y6Bo] or contact uml.scholarlycommunications@manchester.ac.uk providing relevant details, so we can investigate your claim.

\section{OPEN ACCESS}




\title{
Aminoalkyl radicals as halogen-atom transfer agents for activation of alkyl and aryl halides
}

\author{
T. Constantin, ${ }^{1}$ M. Zanini, ${ }^{1}$ A. Regni, ${ }^{1}$ N. S. Sheikh, ${ }^{2}$ F. Juliá ${ }^{1 *}$ and D. Leonori ${ }^{1 *}$
}

${ }^{1}$ School of Chemistry, University of Manchester, Oxford Road, Manchester M13 9PL, UK.

${ }^{2}$ Department of Chemistry, College of Science, King Faisal University, Al-Ahsa 31982, Saudi Arabia

* fabio.juliahernandez@manchester.ac.uk and daniele.leonori@manchester.ac.uk

Abstract: Organic halides are important building blocks in synthesis but their use in (photo)redox chemistry is limited by their low reduction potentials. Halogen-atom transfer still remains the most reliable approach to exploit these substrates in radical processes despite its requirement for hazardous reagents and initiators such as tributyltin hydride. Herein, we demonstrate that $\alpha$ aminoalkyl radicals, easily accessible from simple amines, promote the homolytic activation of carbon-halogen bonds with a reactivity profile mirroring that of classical tin radicals. This strategy conveniently engages alkyl and aryl halides in a wide range of redox transformations to construct $\mathrm{sp}^{3}-\mathrm{sp}^{3}, \quad \mathrm{sp}^{3}-\mathrm{sp}^{2}$ and $\mathrm{sp}^{2}-\mathrm{sp}^{2}$ carbon-carbon bonds under mild conditions with high chemoselectivity.

One Sentence Summary: $\alpha$-Aminoalkyl radicals enable halogen-atom abstraction from unactivated alkyl and aryl halides with a reactivity profile analogous to the one of tin radicals.

Main Text: Carbon radicals are versatile synthetic intermediates central to the preparation of highvalue compounds $(1,2)$. The advent of visible-light photoredox catalysis (3) has offered a broadly applicable radical generation protocol, transforming a variety of redox-active precursors into openshell intermediates by single-electron transfer (SET) and fragmentation (4- 6 ). However, photoredox activation has thus far rarely extended to organic halides, one of the largest class of building blocks available to organic chemists. The current synthetic gap is especially evident in the case of unactivated alkyl halides, where only dehalogenation and intramolecular cyclization of iodides have been reported (7-10). The difficulties in engaging these feedstocks in redox chemistry arise from their highly negative reduction potentials $\left(E_{\text {red }}<-2 \mathrm{~V}\right.$ vs SCE for unactivated alkyl and aryl iodides), which in turn necessitate the use of strongly reducing systems $(11,12)$ (Fig. 1A). Furthermore, the mechanisms involved in photoredox reactions are often uncertain (9), displaying large redox mismatches $(>1 \mathrm{~V})$ for SET activation, which has in turn thwarted the exploitation of the carbon radicals accessed in this manner.

This lack of synthetic applicability stands in stark contrast to the fundamental role alkyl and aryl halides have played in the development of radical chemistry. Methods based on tin/silicon reagents and trialkylboranes- $\mathrm{O}_{2}$ have proven to be highly reliable in accessing carbon radicals from organic halides, generating the open-shell intermediate by homolytic carbon-halogen bond cleavage via 
halogen-atom transfer (XAT) (13-15). However, the toxic, hazardous nature of these reagents and initiators is problematic and has been one of the main drives towards the identification of alternative precursors and chemical strategies for carbon radical generation. Nevertheless, silicon radicals has been recently used in metallaphotoredox catalysis to overcome sluggish carbonhalogen oxidative additions with transition metals $(16,17)$.

We questioned whether $\alpha$-aminoalkyl radicals could serve as a distinct class of halogenabstracting reagents (Fig. 1B). Our idea for this reactivity stemmed from the fact that although classical XAT processes benefit from the formation of strong halogen-tin/silicon bonds, it is the high degree of charge-transfer in the transition state that facilitates halogen-atom abstraction by these nucleophilic radicals (18). We therefore reasoned that strongly nucleophilic $\alpha$-aminoalkyl radicals might benefit from related kinetic polar effects and manifest the same reactivity. Such radicals can be easily generated from simple amines, a class of abundant and inexpensive reagents that would offer ample opportunity for fine steric and electronic tuning.

Here, we report the successful realization of this concept and its implementation as part of a mild and general strategy for the engagement of unactivated alkyl and aryl halides in redox chemistry (Fig. 1C). As $\alpha$-aminoalkyl radicals display a reactivity profile similar to that of tin radicals, their capacity to abstract iodine and bromine atoms has enabled the development of deuteration, crosselectrophile coupling, Heck-type olefination and aromatic $\mathrm{C}-\mathrm{H}$ alkylation protocols.
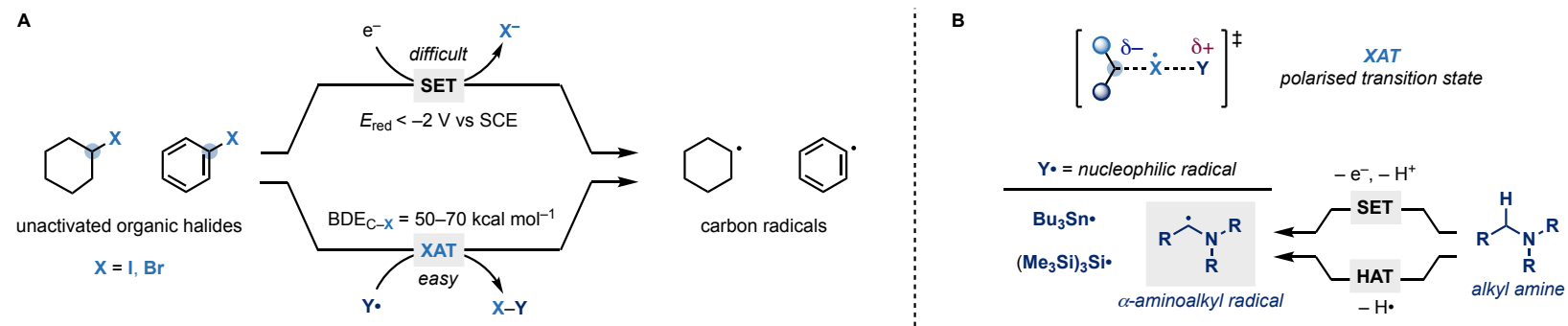

c
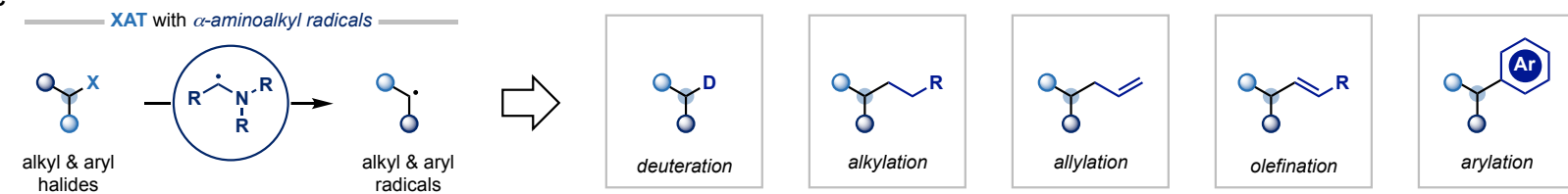

Figure 1. Homolysis of carbon-halogen bonds by $\boldsymbol{\alpha}$-aminoalkyl radicals. (A) Activation modes for the generation of carbon radicals from alkyl and aryl halides. (B) Nucleophilic $\alpha$-aminoalkyl radical abstracts halogen atoms $(\mathrm{X})$ through polarized transition states in analogy to tin and silicon radicals. (C) Outline of the transformations possible using alkyl and aryl halides activated via $\alpha$ aminoalkyl radical-mediated XAT.

We initiated our study by evaluating the iodine-atom transfer reaction from cyclohexyl iodide 2 to the $\alpha$-aminoalkyl radical I-a, derived from triethylamine $\left(\mathrm{Et}_{3} \mathrm{~N}, 1 \mathrm{1a}\right)$ (Fig. 2A). Density functional theory (DFT) calculations predicted this XAT to be kinetically feasible, involving a polarized transition state with a notable charge-transfer character $\left(\delta^{\mathrm{TS}}=0.42\right)$, which supports the anticipated 
interplay of polar effects. Although the XAT is only slightly exothermic,(19) it is the fast and irreversible dissociation of the resulting $\alpha$-iodo-amine III-a into the iminium iodide IV-a that provides the thermodynamic driving force to the process. To gather direct experimental evidence, we generated and monitored I-a using laser flash photolysis $(20,21)$ and observed a noticeable reactivity towards 2 . Data analysis provided a fast rate constant $\left(k_{\mathrm{XAT}}=3.610^{8} \mathrm{M}^{-1} \mathrm{~s}^{-1}\right)$ that is just one order of magnitude slower than reported rates for I-abstraction by $\mathrm{Bu}_{3} \mathrm{Sn} \bullet$ and $\left(\mathrm{Me}_{3} \mathrm{Si}\right)_{3} \mathrm{Si}$ • $\left(\sim 10^{9} \mathrm{M}^{-1} \mathrm{~s}^{-1}\right)(22)$, showing promising potential for implementation in synthetic radical chemistry. To explore the applicability of this strategy in radical reactions, we chose the dehalogenation of 4iodo- $N$-Boc-piperidine 3 using $\mathrm{Et}_{3} \mathrm{~N}$ as XAT-agent precursor and methyl thioglycolate- $\mathrm{H}_{2} \mathrm{O}$ as the $\mathrm{H}$-atom donor (Fig. 2B). At the outset, we were particularly interested to evaluate if the various modes for $\alpha$-amino-radical generation, photochemical or thermal, could be recruited for XAT reactivity. We therefore started by testing four known systems based on amine SET oxidation $\left(\mathrm{Et}_{3} \mathrm{~N}: E_{\mathrm{ox}}=+0.77 \mathrm{~V}\right.$ vs SCE$)$ followed by deprotonation (i.e. photoredox catalysis (23), triplet benzophenone (24) and $\left.\mathrm{SO}_{4}^{--}(25)\right)$ or direct $\mathrm{H}$-atom transfer (HAT) $\left(\mathrm{Et}_{3} \mathrm{~N}: \alpha-\mathrm{N}-\mathrm{C}-\mathrm{H}\right.$ BDE $=91$ $\mathrm{kcal} \mathrm{mol}^{-1}$ ) using $t$-BuO $(26)$. The desired product 4 was obtained in all cases in excellent to good yields, exemplifying the ample variety of conditions for $\alpha$-amino-radical generation and ensuing XAT.

The proposed mechanism under photoredox conditions is depicted in Fig. 2C. Upon blue light irradiation, the excited organic photocatalyst $4 \mathrm{CzIPN}\left({ }^{*} E_{\text {red }}=+1.35 \mathrm{~V}\right.$ vs SCE$)$ oxidizes $1 \mathbf{a}$ which, after subsequent deprotonation, furnishes the key $\alpha$-aminoalkyl radical I-a. This species undergoes XAT with $\mathbf{3}$ and the resulting alkyl radical $\mathbf{V}$ provides the product $\mathbf{4}$ by a favorable HAT from methyl thioglycolate $\left(\mathrm{S}-\mathrm{H} \mathrm{BDE}=87 \mathrm{kcal} \mathrm{mol}^{-1}\right)$. Lastly, SET between the thiyl radical and $4 \mathrm{CzIPN}^{-}$, followed by protonation with $\mathrm{H}_{2} \mathrm{O}$, regenerate the thiol along with the ground-state photocatalyst. The choice of $4 \mathrm{CzIPN}$ and $\mathrm{Et}_{3} \mathrm{~N}$ is relevant to our mechanistic hypothesis because neither the excited nor the reduced state of the photocatalyst $\left(* E_{\mathrm{ox}}=-1.04 \mathrm{~V} ; E_{\mathrm{red}}=-1.21 \mathrm{~V}\right.$ vs $\operatorname{SCE}(27))$ nor I-a $\left(E_{\text {ox }}=-1.12 \mathrm{~V}\right.$ vs SCE (26)) are strong enough to promote direct SET reduction of $3\left(E_{\text {red }}=-2.35 \mathrm{~V}\right.$ vs SCE). This means that the carbon-radical generation is now dissected by the redox requirements of the system and therefore the reductive ability of the photocatalyst is not crucial to the outcome of the reaction. Indeed, this process can be achieved with a diverse range of photocatalysts including those of limited reductive power (e.g. Fukuzumi's acridinium; ${ }^{*} E_{\mathrm{ox}}=$ $-0.57 \mathrm{~V}$ vs SCE). The replacement of $\mathrm{Et}_{3} \mathrm{~N}$ with other common electron donors (e.g. $\mathrm{Ph}_{2} \mathrm{~N}(\mathrm{PMP})$, sodium ascorbate or Hantzsch ester) suppressed the reactivity, despite all effectively quenching the excited photocatalyst.(19) Moreover, other alkyl amines were tested but crucially only those able to generate an $\alpha$-aminoalkyl radical promoted the desired reactivity.(19) These results suggest alkyl iodide activation via a reductive-quenching photoredox cycle is not operative and that the amine plays a fundamental role in the $\mathrm{C}-\mathrm{I}$ bond cleavage that goes beyond its capacity to act as an electron donor.

The high yields obtained with the photoredox system along with the use of $\mathrm{H}_{2} \mathrm{O}$ as stoichiometric $\mathrm{H}$-atom source prompted exploration of dehalogenation-deuteration reactions using $\mathrm{D}_{2} \mathrm{O}$ (Fig. 2D). After optimization, we achieved efficient deuteration of primary, secondary and tertiary alkyl 
iodides in nearly quantitively yields (5-12). The mild reaction conditions tolerated multiple functional groups showcasing the strong chemoselectivity of this XAT approach. Activation of alkyl bromides is still a challenging task in radical chemistry and, for example, it is considered unfeasible using trialkyl borane- $\mathrm{O}_{2}$ systems (28). We were pleased to see that our $\alpha$-aminoalkyl radical-based XAT strategy was applicable to bromides albeit in lower conversion compared to the iodides.

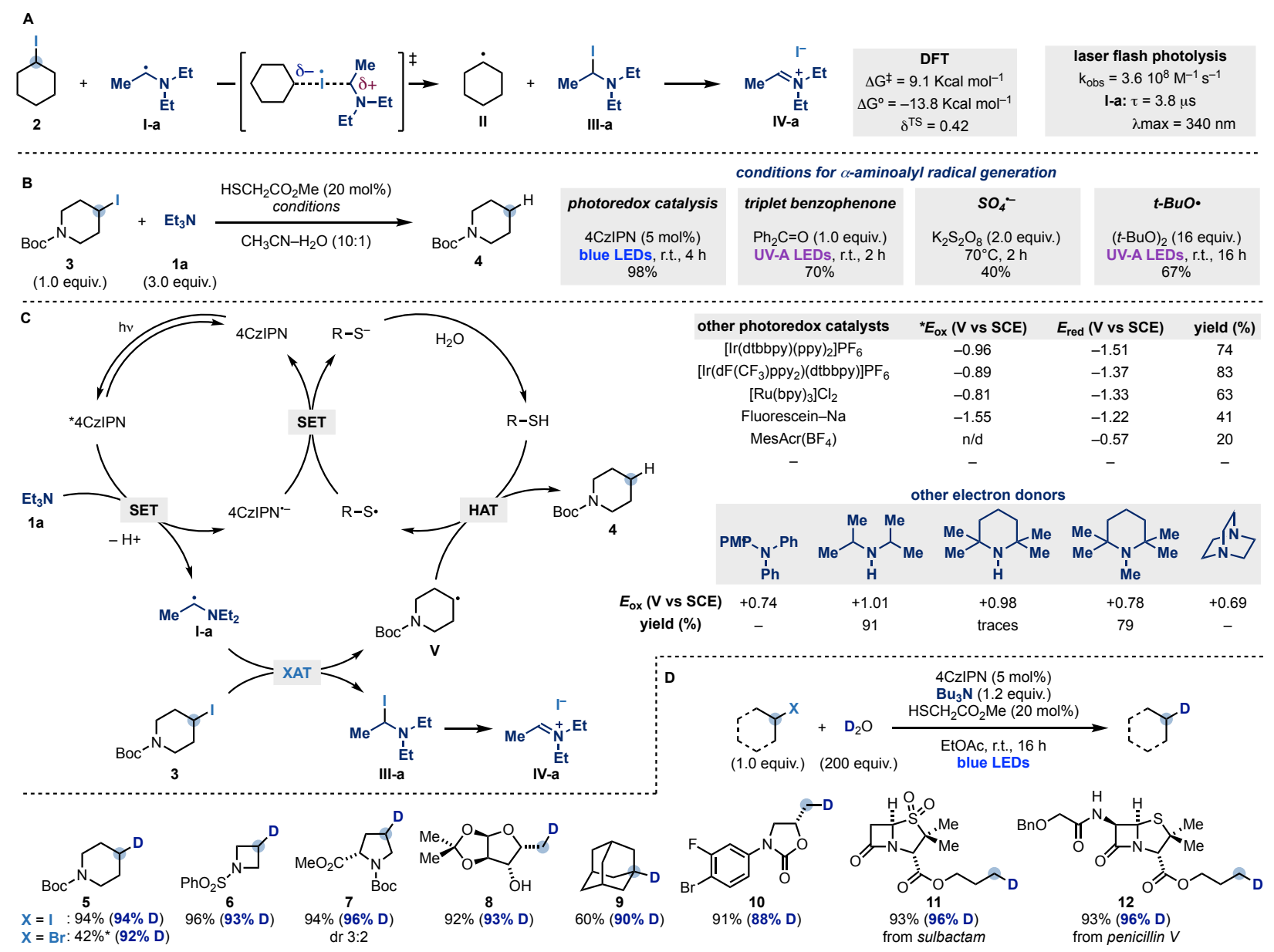

Figure 2. Mechanistic analysis and application to dehalogenation and deuteration reactions. (A) Computational [B3LYP-D3/def2-TZVP] and laser flash photolysis studies on a model XAT reaction with an alkyl iodide. (B) Evaluation of photochemical and thermal strategies for $\alpha$ aminoalkyl radical generation and their use in the dehalogenation of alkyl iodide 3. (B) Proposed mechanism for the photoredox-based dehalogenation of alkyl iodide 3. Mechanistic studies support the intermediacy of a $\alpha$-aminoalkyl radical in the activation of the $\mathrm{C}-\mathrm{I}$ bond. (C) Application of the XAT methodology in deuteration of alkyl halides. All yields are isolated. Deuteration determined by GC-MS/quantitative ${ }^{13} \mathrm{C}$ NMR spectroscopy. * Tribenzylamine $\mathbf{1 b}$ was used as the amine. r. t., room temperature. 
The XAT strategy generates carbon radicals from organic halides oxidatively, which represents an umpolung approach relative to the natural redox requirement for SET activation of these building blocks. We posited that the generated radicals could therefore be used in similar mechanistic scenarios to carboxylic acids or potassium trifluoroborates, allowing their modular application in net reductive processes, such as cross-electrophile couplings $(29,30)$.

We explored this premise by developing Giese-type hydroalkylation of electron-poor olefins. Although these transformations have been performed with the aid of nickel catalysis, they typically require the use of stoichiometric metal reductants (e.g. $\mathrm{Mn}^{0}, \mathrm{Zn}^{0}$ ) or silane H-donors $(31,32)$. In our case, as $\alpha$-aminoalkyl radicals have been used as substrates in Giese additions (33), the success of this strategy hinged on their capacity to undergo preferential XAT over their known reaction with the olefin. Exploration began with 3-iodo- $N$-Boc-azetidine in the presence of $\mathrm{Et}_{3} \mathrm{~N}$ and 4CzIPN under blue light irradiation (Fig. 3A see Fig. S10 for a proposed mechanism). A diverse range of electron poor olefins were efficiently converted into the corresponding products in high to excellent yields (13-23). A variety of functionalities were readily accommodated including polar groups such as free carboxylic acid, primary amide, pyridine and boronic ester. When the same reactions were attempted using 3-bromo- $N$-Boc-azetidine, no desired product was obtained and a significant amount of the adduct arising from the direct addition of $\mathbf{I}-\mathbf{a}$ to the olefin acceptor was identified (Fig. 3B). In this case, owing to the stronger nature of the $\mathrm{C}-\mathrm{Br}$ bond, XAT is slower thus rendering the direct Giese reaction of $\mathbf{I}-\mathbf{a}$ with the acceptor competitive $\left(k_{\mathrm{obs}} \sim 10^{7} \mathrm{M}^{-1} \mathrm{~s}^{-1}\right.$ (21)). We therefore reasoned that the modulation of the electronic and steric properties of the $\alpha$ aminoalkyl radical could be used to tune its reactivity. Indeed, by using tribenzylamine (1b) we restored XAT as the favored pathway for reactions of unactivated alkyl bromides in these hydroalkylations. As the stabilized $\alpha$-aminoalkyl radical I-b was essentially unreactive towards electron poor olefins $\left(k_{\text {calc }} \sim 10^{-1} \mathrm{M}^{-1} \mathrm{~s}^{-1}(21)\right)$, bromine abstraction was now possible providing the desired products in good yields.

We next explored the alkyl iodide scope using Boc-protected dehydroalanine as olefin acceptor, providing convenient access to unnatural amino acids (24-35). Also in this case, a wide variety of organyl groups bearing common functionalities such as free alcohol, alkyl chloride, silane and terminal alkyne were compatible, reflecting the mildness of the reaction conditions. Furthermore, this protocol has also been carried out at gram-scale without erosion in yield. The ability to generate primary alkyl radicals complements approaches using oxalates and trifluoroborates which are known to suffer from sluggish fragmentations $(34,35)$. When alkyl halides activated towards $\mathrm{S}_{\mathrm{N}} 2$ attack by $\mathrm{Et}_{3} \mathrm{~N}$ were employed (e.g. 29 and 32), not surprisingly the desired products were obtained in low yields. This hurdle was addressed by adjusting the steric properties of the XATreagent: using the bulkier amine 1,2,2,6,6-pentamethylpiperidine (1c), efficient couplings were achieved. We have also been able to extend this methodology to unactivated aryl iodides using the more hindered but less stabilized $\alpha$-aminoalkyl radical derived from triisobutylamine (1d). These conditions enabled direct access to aryl radicals by $\mathrm{sp}^{2} \mathrm{C}-\mathrm{I}$ bond cleavage and were applied to the one-pot transformation of tosylated serine into phenylalanine derivatives (36-39). Overall, these results illustrate how the large structural diversity of available tertiary amines facilitates the 
rational tailoring of the $\alpha$-aminoalkyl radical reactivity to address different challenges in carbonhalogen bond activation.

The XAT strategy for cross-electrophile coupling is not restricted to electron poor olefins. We also achieved efficient allylation of alkyl/aryl halides using simple allyl chlorides and other pseudohalides (40-50) (Fig. 3C, see Fig. S12 for a proposed mechanism). This approach bypasses the conventional conversion of one of the two coupling partners into a Grignard/organozinc reagent (36) and therefore tolerates functionalities, such as free alcohol and ketone, that are often troublesome with organometallics.

A
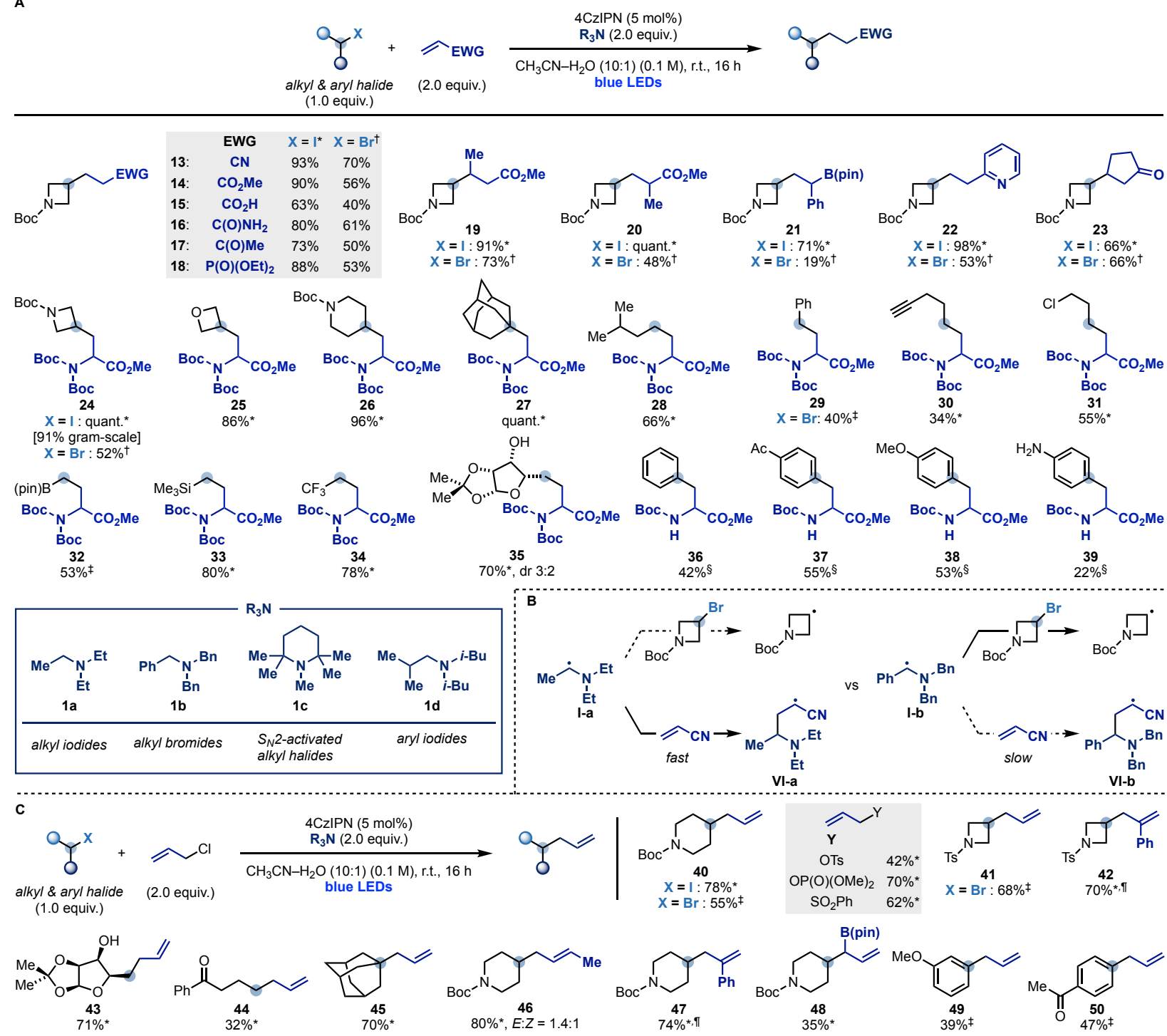

MeO

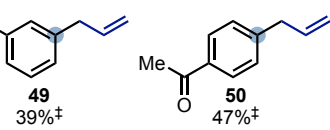

Figure 3. Application to hydroalkylation and allylation. (A) Scope for the alkylation of alkyl iodides, alkyl bromides and aryl iodides. (B) Tailoring XAT reactivity by modifying the $\alpha$ aminoalkyl radical structure. (C) Scope for the allylation of alkyl iodides, alkyl bromides and aryl iodides. All yields are isolated. ${ }^{*} \mathbf{1 a}$ was used as the amine. ${ }^{\dagger} \mathbf{1 b}$ was used as the amine. ${ }^{\ddagger} \mathbf{1 c}$ was used as the amine. ${ }^{\S} \mathbf{1 d}$ was used as the amine. ${ }^{\pi}$ The corresponding allyl sulfone was used. 
To further demonstrate the versatility of this activation mode, we sought to adapt it to target the use of alkyl halides in Heck-type olefinations, a long-standing challenge in conventional palladium catalysis due to undesired $\beta$-hydride-elimination (37-39). Specifically, we questioned whether, after addition of alkyl radicals to suitable olefins (VII), a cobaloxime co-catalyst might trigger a dehydrogenation reaction (40), thus leading to $\mathrm{sp}^{3}-\mathrm{sp}^{2} \mathrm{C}-\mathrm{C}$ bond formation (via VIII) without the need for precious metals (see Fig. S14 for a proposed mechanism). As shown in Fig. 4A, we found this dual XAT- $-\mathrm{Co}$ ] protocol feasible thus allowing the direct olefination of primary, secondary and tertiary alkyl iodides and bromides exclusively as the $E$-isomers (51-74) (with the exception of 54 and 62). The broad functional group compatibility was demonstrated with the successful engagement of substrates containing phenol, aniline and benzoic acid moieties as well as aryl bromide, boronic acid and phosphine groups that could limit application under transition metal catalysis. The olefination was also very effective in intramolecular settings as showcased by the construction of tricyclic $\mathbf{7 5}$ in good yield. Couplings with aryl iodides were attempted but generally resulted in low yields.

A
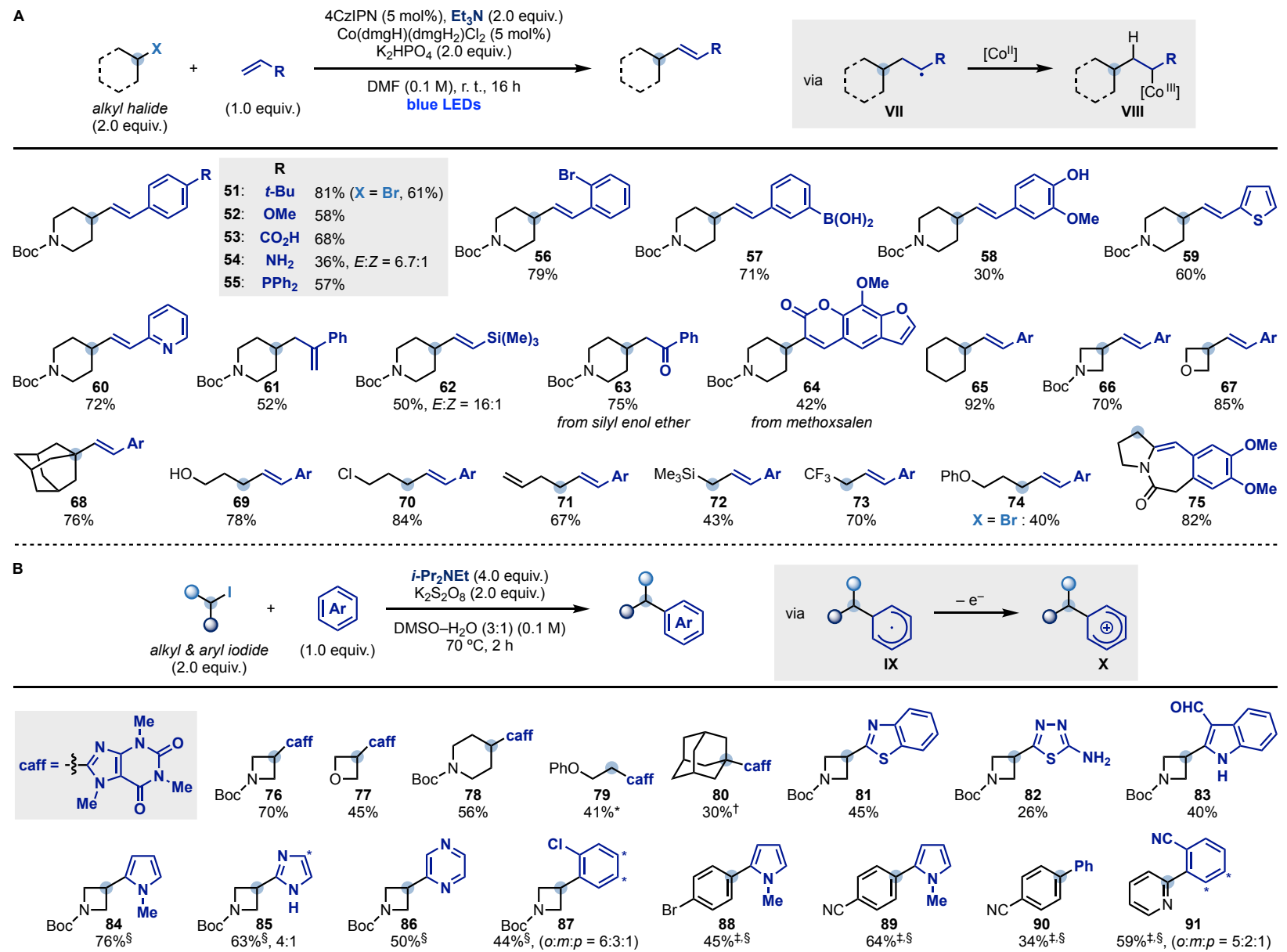

Figure 4. Application to olefinations and arylations. (A) Scope for olefination of alkyl iodides and alkyl bromides. (B) Scope for the $\mathrm{C}-\mathrm{H}$ alkylation and arylation of aromatics. All yields are 
isolated. * $1 \mathrm{c}$ was used as the amine. ${ }^{\dagger} \mathrm{Me}_{3} \mathrm{~N}$ was used as the amine. ${ }^{\ddagger} \mathrm{Bu}_{3} \mathrm{~N}$ was used as the amine. $\S$ The reaction was run with 50 equiv. of the arene.

In a final effort to establish the generality of this XAT strategy, we turned our attention to the direct aromatic C-H alkylation via radical intermediates (Fig. 4B, see Fig. S15 for a proposed mechanism). Recently, the use of zinc-alkylsulfinates has provided a powerful and effective solution to this synthetic challenge $(41,42)$. However, as these reagents are often prepared from the corresponding halides, a methodology that directly used these building blocks would obviate multistep synthesis of any reactive intermediate. In this case however, a photoredox system for $\alpha$ aminoalkyl radical generation is difficult to implement due to the mechanistic requirement of a second oxidation after radical addition to the arene in order to allow re-aromatization (IX $\rightarrow \mathbf{X})$. The broad set of reactivity modes for $\alpha$-aminoalkyl radical generation enabled identification of simple thermal, net oxidative conditions for the direct alkylation of caffeine with alkyl iodides without the need for light or catalysts (76-80). This manifold for aromatic $\mathrm{C}-\mathrm{H}$ alkylation was compatible with the installation of primary, secondary and tertiary alkyl groups and could be extended to other heteroarenes commonly found in bioactive molecules such indole and azoles as well as benzenoids (43) (81-87). Furthermore, we demonstrated that aryl iodide activation and subsequent $\mathrm{sp}^{2}-\mathrm{sp}^{2}$ coupling (44) is also possible, as shown by the successful preparation of 8891.

The results presented here demonstrate that alkyl and aryl halides can be converted into carbonradicals by halogen-atom transfer using $\alpha$-aminoalkyl radicals. We believe that the broad scope, functional group tolerance and modularity of this approach for carbon-halogen bond activation will be of great utility to chemists working in both academia and industry.

Acknowledgments: We gratefully acknowledge Dr Derren Heyes for help with laser flash photolysis studies; Funding: D. L. thanks EPSRC for a Fellowship (EP/P004997/1) and the European Research Council for a research grant (758427); Author contributions: F. J. and D. L. designed the project and wrote the manuscript. T. C., M. Z., A. R. and F. J. performed all the experiments. N. S. S. performed the computational studies. All the authors analyzed the results; Competing interests: Authors declare no competing interests. Data and materials availability: All data are available in the main text or the supplementary materials. 


\section{Supplementary Materials}

Materials and Methods

Figures S1 to S24

Tables S1 to S16

References (45-108)

1. J. M. Smith, S. J. Harwood, P. S. Baran, Radical Retrosynthesis. Acc. Chem. Res. 51, 1807-1817 (2018).

2. S. P. Pitre, N. A. Weires, L. E. Overman, Forging C(sp3)-C(sp3) Bonds with CarbonCentered Radicals in the Synthesis of Complex Molecules. J. Am. Chem. Soc. 141, 28002813 (2019).

3. C. K. Prier, D. A. Rankic, D. W. C. MacMillan, Visible Light Photoredox Catalysis with Transition Metal Complexes: Applications in Organic Synthesis. Chem. Rev. 113, 53225363 (2013).

4. J. Xuan, Z.-G. Zhang, W.-J. Xiao, Visible-Light-Induced Decarboxylative Functionalization of Carboxylic Acids and Their Derivatives. Angew. Chem. Int. Ed. 54, 15632-15641 (2015).

5. J. K. Matsui, S. B. Lang, D. R. Heitz, G. A. Molander, Photoredox-Mediated Routes to Radicals: The Value of Catalytic Radical Generation in Synthetic Methods Development. ACS Catal. 7, 2563-2575 (2017).

6. A. Togni et al., Pyridinium Salts as Redox-Active Functional Group Transfer Reagents. Angew. Chem. Int. Ed., DOI: 10.002/anie.201911660.

7. J. D. Nguyen, E. M. D'Amato, J. M. R. Narayanam, C. R. J. Stephenson, Engaging unactivated alkyl, alkenyl and aryl iodides in visible-light-mediated free radical reactions. Nat. Chem. 4, 854 (2012).

8. H. Kim, C. Lee, Visible-Light-Induced Photocatalytic Reductive Transformations of Organohalides. Angew. Chem. Int. Ed. 51, 12303-12306 (2012).

9. Y. Shen, J. Cornella, F. Juliá-Hernández, R. Martin, Visible-Light-Promoted Atom Transfer Radical Cyclization of Unactivated Alkyl Iodides. ACS Catal. 7, 409-412 (2017).

10. D. Alpers, M. Gallhof, J. Witt, F. Hoffmann, M. Brasholz, A Photoredox-Induced Stereoselective Dearomative Radical (4+2)-Cyclization/1,4-Addition Cascade for the Synthesis of Highly Functionalized Hexahydro-1H-carbazoles. Angew. Chem. Int. Ed. 56, 1402-1406 (2017).

11. J. L. Kuo, C. Lorenc, J. M. Abuyuan, J. R. Norton, Catalysis of Radical Cyclizations from Alkyl Iodides under H2: Evidence for Electron Transfer from [CpV(CO)3H]-. J. Am. Chem. Soc. 140, 4512-4516 (2018).

12. G. Nocera et al., Electron Transfer Reactions: KOtBu (but not NaOtBu) Photoreduces Benzophenone under Activation by Visible Light. J. Am. Chem. Soc. 140, 9751-9757 (2018).

13. W. P. Neumann, Tri-n-butyltin Hydride as Reagent in Organic Synthesis. Synthesis 1987, 665-683 (1987). 
14. C. Chatgilialoglu, C. Ferreri, Y. Landais, V. I. Timokhin, Thirty Years of (TMS)3SiH: A Milestone in Radical-Based Synthetic Chemistry. Chem. Rev. 118, 6516-6572 (2018).

15. H. Yorimitsu, K. Oshima, in Radicals in Organic Synthesis, P. Renaud, M. P. Sibi, Eds. (2008), pp. 11-27.

16. C. Le, T. Q. Chen, T. Liang, P. Zhang, D. W. C. MacMillan, A radical approach to the copper oxidative addition problem: Trifluoromethylation of bromoarenes. Science $\mathbf{3 6 0}$, 1010-1014 (2018).

17. P. Zhang, C. C. Le, D. W. C. MacMillan, Silyl Radical Activation of Alkyl Halides in Metallaphotoredox Catalysis: A Unique Pathway for Cross-Electrophile Coupling. J. Am. Chem. Soc. 138, 8084-8087 (2016).

18. W. H. Tamblyn, E. A. Vogler, J. K. Kochi, Polar effect in alkyl radical reactions. Carbon kinetic isotope effects in halogen atom transfer to tin(III) and chromium(II). J. Org. Chem. 45, 3912-3915 (1980).

19. C. Ollivier, P. Renaud, Organoboranes as a Source of Radicals. Chemical Reviews 101, 3415-3434 (2001).

20. J. C. Scaiano, in Reactive Intermediate Chemistry, R. A. Moss, M. S. Platz, M. Jones, Eds. (2005), pp. 847-871.

21. J. Lalevée, B. Graff, X. Allonas, J. P. Fouassier, Aminoalkyl Radicals: Direct Observation and Reactivity toward Oxygen, 2,2,6,6-Tetramethylpiperidine-N-oxyl, and Methyl Acrylate. J. Phys. Chem. A 111, 6991-6998 (2007).

22. K. U. Ingold, J. Lusztyk, J. C. Scaiano, Absolute rate constants for the reactions of tributylgermyl and tributylstannyl radicals with carbonyl compounds, other unsaturated molecules, and organic halides. J. Am. Chem. Soc. 106, 343-348 (1984).

23. K. Nakajima, Y. Miyake, Y. Nishibayashi, Synthetic Utilization of $\alpha$-Aminoalkyl

Radicals and Related Species in Visible Light Photoredox Catalysis. Acc. Chem. Res. 49, 1946-1956 (2016).

24. S. G. Cohen, A. Parola, G. H. Parsons, Photoreduction by amines. Chem. Rev. 73, 141161 (1973).

25. J. K. Laha, K. S. S. Tummalapalli, A. Nair, N. Patel, Sulfate Radical Anion (SO4•-) Mediated C(sp3)-H Nitrogenation/Oxygenation in N-Aryl Benzylic Amines Expanded the Scope for the Synthesis of Benzamidine/Oxazine Heterocycles. J. Org. Chem. 80, 11351-11359 (2015).

26. D. D. M. Wayner, J. J. Dannenberg, D. Griller, Oxidation potentials of $\alpha$-aminoalkyl radicals: bond dissociation energies for related radical cations. Chem. Phys. Lett. 131, 189-191 (1986).

27. J. Luo, J. zhang, Donor-Acceptor Fluorophores for Visible-Light-Promoted Organic Synthesis: Photoredox/Ni Dual Catalytic C(sp3)-C(sp2) Cross-Coupling. ACS Catal. 6, 873-877 (2016).

28. V. Soulard, G. Villa, D. P. Vollmar, P. Renaud, Radical Deuteration with D2O: Catalysis and Mechanistic Insights. J. Am. Chem. Soc. 140, 155-158 (2018).

29. D. A. Everson, D. J. Weix, Cross-Electrophile Coupling: Principles of Reactivity and Selectivity. J. Org. Chem. 79, 4793-4798 (2014).

30. E. Richmond, J. Moran, Recent Advances in Nickel Catalysis Enabled by Stoichiometric Metallic Reducing Agents. Synthesis 50, 499-513 (2018). 
31. K. M. M. Huihui, R. Shrestha, D. J. Weix, Nickel-Catalyzed Reductive Conjugate Addition of Primary Alkyl Bromides to Enones To Form Silyl Enol Ethers. Organic Letters 19, 340-343 (2017).

32. F. Zhou, J. Zhu, Y. Zhang, S. Zhu, NiH-Catalyzed Reductive Relay Hydroalkylation: A Strategy for the Remote C(sp3)-H Alkylation of Alkenes. Angew. Chem. Int. Ed. 130, 4122-4126 (2018).

33. R. A. Aycock, C. J. Pratt, N. T. Jui, Aminoalkyl Radicals as Powerful Intermediates for the Synthesis of Unnatural Amino Acids and Peptides. ACS Catal. 8, 9115-9119 (2018).

34. C. C. Nawrat, C. R. Jamison, Y. Slutskyy, D. W. C. MacMillan, L. E. Overman, Oxalates as Activating Groups for Alcohols in Visible Light Photoredox Catalysis: Formation of Quaternary Centers by Redox-Neutral Fragment Coupling. J. Am. Chem. Soc. 137, 11270-11273 (2015).

35. C. Lévêque, L. Chenneberg, V. Corcé, C. Ollivier, L. Fensterbank, Organic photoredox catalysis for the oxidation of silicates: applications in radical synthesis and dual catalysis. Chem. Commun. 52, 9877-9880 (2016).

36. H. Yorimitsu, K. Oshima, Recent Progress in Asymmetric Allylic Substitutions Catalyzed by Chiral Copper Complexes. Angew. Chem. Int. Ed. 44, 4435-4439 (2005).

37. M. R. Netherton, G. C. Fu, in Palladium in Organic Synthesis: -/-, J. Tsuji, Ed. (Springer Berlin Heidelberg, Berlin, Heidelberg, 2005), pp. 85-108.

38. G.-Z. Wang, R. Shang, W.-M. Cheng, Y. Fu, Irradiation-Induced Heck Reaction of Unactivated Alkyl Halides at Room Temperature. J. Am. Chem. Soc. 139, 18307-18312 (2017).

39. D. Kurandina, M. Parasram, V. Gevorgyan, Visible Light-Induced Room-Temperature Heck Reaction of Functionalized Alkyl Halides with Vinyl Arenes/Heteroarenes. Angew. Chem. Int. Ed. 56, 14212-14216 (2017).

40. X. Sun, J. Chen, T. Ritter, Catalytic dehydrogenative decarboxyolefination of carboxylic acids. Nat. Chem. 10, 1229-1233 (2018).

41. Y. Fujiwara et al., Practical and innate carbon-hydrogen functionalization of heterocycles. Nature 492, 95 (2012).

42. R. Gianatassio et al., Simple Sulfinate Synthesis Enables C H Trifluoromethylcyclopropanation. Angew. Chem. Int. Ed. 53, 9851-9855 (2014).

43. Z. Jiao, L. H. Lim, H. Hirao, J. S. Zhou, Palladium-Catalyzed para-Selective Alkylation of Electron-Deficient Arenes. Angew. Chem. Int. Ed. 57, 6294-6298 (2018).

44. I. Ghosh, T. Ghosh, J. I. Bardagi, B. König, Reduction of aryl halides by consecutive visible light-induced electron transfer processes. Science 346, 725-728 (2014).

45. E. D. Nacsa, D. W. C. MacMillan, Spin-Center Shift-Enabled Direct Enantioselective $\alpha$ Benzylation of Aldehydes with Alcohols. J. Am. Chem. Soc. 140, 3322-3330 (2018).

46. H. J. Kuhn, S. E. Braslavsky, R. Schmidt, Chemical actinometry (IUPAC technical report). Pure Appl. Chem. 76 (2004), pp. 2105-2146.

47. Y. Y. Loh, K. Nagao, A. J. Hoover, D. Hesk, N. R. Rivera, S. L. Colletti, I. W. Davies, D. W. C. MacMillan, Photoredox-catalyzed deuteration and tritiation of pharmaceutical compounds. Science (80-. ). 358, 1182-1187 (2017).

48. J. Luo, J. Zhang, Donor-Acceptor Fluorophores for Visible-Light-Promoted Organic Synthesis: Photoredox/Ni Dual Catalytic C(sp3)-C(sp2) Cross-Coupling. ACS Catal. 6, 873-877 (2016). 
49. C. K. Prier, D. A. Rankic, D. W. C. MacMillan, Visible Light Photoredox Catalysis with Transition Metal Complexes: Applications in Organic Synthesis. Chem. Rev. 113, 53225363 (2013).

50. N. A. Romero, D. A. Nicewicz, Organic Photoredox Catalysis. Chem. Rev. 116, 1007510166 (2016).

51. S. L. J. Tan, R. D. Webster, Electrochemically Induced Chemically Reversible ProtonCoupled Electron Transfer Reactions of Riboflavin (Vitamin B 2 ). J. Am. Chem. Soc. 134, 5954-5964 (2012).

52. M. J. Sevrin, L. Furst, J. D. Nguyen, J. L. Collins, C. R. J. Stephenson, Lithium bis-catechol borate as an effective reductive quencher in photoredox catalysis. Tetrahedron. 74, 32463252 (2018).

53. Merck Photocatalysis Chart, (available at http://chemlabs.princeton.edu/macmillan/wpcontent/uploads/sites/6/Merck-Photocatalysis-Chart.pdf).

54. K. N. Lee, Z. Lei, M. Y. Ngai, $\beta$-Selective Reductive Coupling of Alkenylpyridines with Aldehydes and Imines via Synergistic Lewis Acid/Photoredox Catalysis. J. Am. Chem. Soc. 139, 5003-5006 (2017).

55. J. L. Jeffrey, F. R. Petronijević, D. W. C. Macmillan, Selective Radical-Radical CrossCouplings: Design of a Formal $\beta$-Mannich Reaction. J. Am. Chem. Soc. 137, 8404-8407 (2015).

56. D. Griller, J. A. Howard, P. R. Marriott, J. C. Scaiano, Absolute Rate Constants for the Reactions of tert-Butoxyl, tert-Butylperoxyl, and Benzophenone Triplet with Amines: The Importance of a Stereoelectronic Effect. J. Am. Chem. Soc. 103, 619-623 (1981).

57. Z. R. Zheng, D. H. Evans, S. F. Nelsen, Studies of the anodic oxidation of 1,4diazabicyclo[2.2.2] octane. Reactions of the radical cation. J. Org. Chem. 65, 1793-1798 (2000).

58. R. Ishimatsu, S. Matsunami, K. Shizu, C. Adachi, K. Nakano, T. Imato, Solvent effect on thermally activated delayed fluorescence by 1,2,3,5-tetrakis(carbazol-9-yl)-4,6dicyanobenzene. J. Phys. Chem. A. 117, 5607-5612 (2013).

59. V. Soulard, G. Villa, D. P. Vollmar, P. Renaud, Radical Deuteration with D 2 O: Catalysis and Mechanistic Insights. J. Am. Chem. Soc. 140, 155-158 (2018).

60. G. A. Olah, P. Ramaiah, C. B. Rao, G. Sandford, R. Golam, N. J. Trivedi, J. A. Olah, Nitration of Adamantane and Diamantane with Nitronium Tetrafluoroborate. J. Am. Chem. Soc. 115, 7246-7249 (1993).

61. Z. Liu, S. Wei, A. Liang, J. Li, D. Zou, Y. Wu, Y. Wu, Palladium-catalyzed reductive Heck reaction of $\alpha, \beta$-unsaturated alkenes and cycloalkyl iodides. Tetrahedron Lett. 60, 485-488 (2019).

62. M. Tuyishime, R. Lawrence, S. Cocklin, Core chemotype diversification in the HIV-1 entry inhibitor class using field-based bioisosteric replacement. Bioorganic Med. Chem. Lett. 26, 228-234 (2016).

63. M. Silvi, V. K. Aggarwal, Radical Addition to Strained $\sigma$-Bonds Enables the Stereocontrolled Synthesis of Cyclobutyl Boronic Esters. J. Am. Chem. Soc. 141, 95119515 (2019).

64. A. Fawcett, J. Pradeilles, Y. Wang, T. Mutsuga, E. L. Myers, V. K. Aggarwal, Photoinduced decarboxylative borylation of carboxylic acids. Science (80-. ). 357, 283-286 (2017).

65. J. A. Shin, J. Kim, H. Lee, S. Ha, H. Y. Lee, Cu(OTf)2-Promoted 1,4-addition of alkyl bromides to dehydroalanine. J. Org. Chem. 84, 4558-4565 (2019). 
66. B. Basu, M. M. H. Bhuiyan, P. Das, I. Hossain, Catalytic transfer reduction of conjugated alkenes and an imine using polymer-supported formates. Tetrahedron Lett. 44, 8931-8934 (2003).

67. J. Lalevée, B. Graff, X. Allonas, J. P. Fouassier, Aminoalkyl radicals: Direct observation and reactivity toward oxygen, 2,2,6,6-tetramethylpiperidine-N-oxyl, and methyl acrylate. $J$. Phys. Chem. A. 111, 6991-6998 (2007).

68. L. Gonnard, A. Guérinot, J. Cossy, Cobalt-Catalyzed Cross-Coupling of 3- and 4Iodopiperidines with Grignard Reagents. Chem. - A Eur. J. 21, 12797-12803 (2015).

69. R. Gianatassio, D. Kadish, Direct alkylation of 1-azabicyclo[1.1.0]butanes. Org. Lett. 21, 2060-2063 (2019).

70. S. Achab, B. C. Das, A concise and general entry into (R)-4-hydroxy-2-substituted cyclopent2-enones from D-glucose: Chiral intermediates for the synthesis of PGE2, ( -)pentenomycin I, and allethrin. J. Chem. Soc. Perkin Trans. 1, 2863-2873 (1990).

71. J. J. Cao, F. Zhou, J. Zhou, Improving the atom efficiency of the wittig reaction by a "waste as catalyst/Co-catalyst" strategy. Angew. Chemie - Int. Ed. 49, 4976-4980 (2010).

72. G. Z. Wang, J. Jiang, X. S. Bu, J. J. Dai, J. Xu, Y. Fu, H. J. Xu, Copper-Catalyzed CrossCoupling Reaction of Allyl Boron Ester with $1^{\circ} / 2^{\circ} / 3^{\circ}$-Halogenated Alkanes. Org. Lett. 17, 3682-3685 (2015).

73. F. Zhou, J. Zhu, Y. Zhang, S. Zhu, NiH-Catalyzed Reductive Relay Hydroalkylation: A Strategy for the Remote C(sp3)-H Alkylation of Alkenes. Angew. Chemie - Int. Ed. 57, 4058-4062 (2018).

74. M. Lafrance, M. Roggen, E. M. Carreira, Direct, enantioselective iridium-catalyzed allylic amination of racemic allylic alcohols. Angew. Chemie - Int. Ed. 51, 3470-3473 (2012).

75. E. Alacid, C. Nájera, Palladium-catalyzed cross-coupling reactions of potassium alkenyltrifluoroborates with organic halides in aqueous media. J. Org. Chem. 74, 23212327 (2009).

76. M. O. Akram, P. S. Mali, N. T. Patil, Cross-Coupling Reactions of Aryldiazonium Salts with Allylsilanes under Merged Gold/Visible-Light Photoredox Catalysis. Org. Lett. 19, 3075-3078 (2017).

77. M. Koy, F. Sandfort, A. Tlahuext-Aca, L. Quach, C. G. Daniliuc, F. Glorius, PalladiumCatalyzed Decarboxylative Heck-Type Coupling of Activated Aliphatic Carboxylic Acids Enabled by Visible Light. Chem. - A Eur. J. 24, 4552-4555 (2018).

78. J. Wu, P. S. Grant, X. Li, A. Noble, V. K. Aggarwal, Catalyst-Free Deaminative Functionalizations of Primary Amines by Photoinduced Single-Electron Transfer. Angew. Chemie - Int. Ed. 58, 5697-5701 (2019).

79. A. Chatupheeraphat, H. H. Liao, W. Srimontree, L. Guo, Y. Minenkov, A. Poater, L. Cavallo, M. Rueping, Ligand-Controlled Chemoselective C(acyl)-O Bond vs C(aryl)-C Bond Activation of Aromatic Esters in Nickel Catalyzed C(sp2)-C(sp3) Cross-Couplings. J. Am. Chem. Soc. 140, 3724-3735 (2018).

80. G. Z. Wang, R. Shang, Y. Fu, Irradiation-Induced Palladium-Catalyzed Decarboxylative Heck Reaction of Aliphatic N-(Acyloxy)phthalimides at Room Temperature. Org. Lett. 20, 888-891 (2018).

81. Z. H. Xia, C. L. Zhang, Z. H. Gao, S. Ye, Switchable Decarboxylative Heck-Type Reaction and Oxo-alkylation of Styrenes with N-Hydroxyphthalimide Esters under Photocatalysis. Org. Lett. 20, 3496-3499 (2018).

82. X. Wang, Y. Ye, S. Zhang, J. Feng, Y. Xu, Y. Zhang, J. Wang, Copper-catalyzed C(sp 3)- 
C(sp 3) bond formation using a hypervalent iodine reagent: An efficient allylic trifluoromethylation. J. Am. Chem. Soc. 133, 16410-16413 (2011).

83. R. Gianatassio, S. Kawamura, C. L. Eprile, K. Foo, J. Ge, A. C. Burns, M. R. Collins, P. S. Baran, Simple sulfinate synthesis enables C-H trifluoromethylcyclopropanation. Angew. Chemie - Int. Ed. 53, 9851-9855 (2014).

84. J. Dong, Z. Wang, X. Wang, H. Song, Y. Liu, Q. Wang, Metal-, Photocatalyst-, and LightFree Minisci C-H Alkylation of N-Heteroarenes with Oxalates. J. Org. Chem. 84, 75327540 (2019).

85. J. Koeller, P. Gandeepan, L. Ackermann, Visible-Light-Induced Decarboxylative C-H Adamantylation of Azoles at Ambient Temperature. Synth. 51, 1284-1292 (2019).

86. M. A. J. Duncton, M. A. Estiarte, R. J. Johnson, M. Cox, D. J. R. O’Mahony, W. T. Edwards, M. G. Kelly, Preparation of heteroaryloxetanes and heteroarylazetidines by use of a Minisci reaction. J. Org. Chem. 74, 6354-6357 (2009).

87. Y. X. Liu, D. Xue, J. Di Wang, C. J. Zhao, Q. Z. Zou, C. Wang, J. Xiao, Room-temperature arylation of arenes and heteroarenes with diaryliodonium salts by photoredox catalysis. Synlett. 24, 507-513 (2013).

88. G. Sun, S. Ren, X. Zhu, M. Huang, Y. Wan, Direct arylation of pyrroles via indirect electroreductive $\mathrm{C}-\mathrm{H}$ functionalization using perylene bisimide as an electron-transfer mediator. Org. Lett. 18, 544-547 (2016).

89. C. Zhou, J. Wang, L. Li, R. Wang, M. Hong, A palladium chelating complex of ionic watersoluble nitrogen-containing ligand: The efficient precatalyst for Suzuki-Miyaura reaction in water. Green Chem. 13, 2100-2106 (2011).

90. W. C. Chen, Y. C. Hsu, W. C. Shih, C. Y. Lee, W. H. Chuang, Y. F. Tsai, P. P. Y. Chen, T. G. Ong, Metal-free arylation of benzene and pyridine promoted by amino-linked nitrogen heterocyclic carbenes. Chem. Commun. 48, 6702-6704 (2012).

91. J. Lalevée, X. Allonas, J. P. Fouassier, Halogen abstraction reaction between aminoalkyl radicals and alkyl halides: Unusual high rate constants. Chem. Phys. Lett. 454, 415-418 (2008).

92. C. D. McTiernan, S. P. Pitre, J. C. Scaiano, Photocatalytic dehalogenation of vicinal dibromo compounds utilizing sexithiophene and visible-light irradiation. ACS Catal. 4, 4034-4039 (2014).

93. J. C. Scaiano, in Reactive Intermediate Chemistry (John Wiley \& Sons, Inc., Hoboken, NJ, USA, 2005; http://doi.wiley.com/10.1002/0471721492.ch18), pp. 847-871.

94. V. V. Pavlishchuk, A. W. Addison, Conversion constants for redox potentials measured versus different reference electrodes in acetonitrile solutions at $25^{\circ} \mathrm{C}$. Inorganica Chim. Acta. 298, 97-102 (2000).

95. H. G. Roth, N. A. Romero, D. A. Nicewicz, Experimental and Calculated Electrochemical Potentials of Common Organic Molecules for Applications to Single-Electron Redox Chemistry. Synlett. 27, 714-723 (2016).

96. W. Y. R. G. Parr, Density-funcional theory of atoms and molecules (1989).

97. D. J. F. M. J. Frisch, G. W. Trucks, H. B. Schlegel, G. E. Scuseria, M. A. Robb, J. R. Cheeseman, G. Scalmani, V. Barone, G. A. Petersson, H. Nakatsuji, X. Li, M. Caricato, A. Marenich, J. Bloino, B. G. Janesko, R. Gomperts, B. Mennucci, C. Adamo, R. Cammi, J. W., Gaussian 09, Revision A.02 (2016).

98. J. Dennington, R., Keith, T. and Millam, Gauss View. Version 5, Semichem Inc., Shawnee Mission (2009). 
99. A. D. Becke, A new mixing of Hartree-Fock and local density-functional theories. J. Chem. Phys. 98, 1372-1377 (1993).

100. A. D. Becke, Density-functional thermochemistry. III. The role of exact exchange. J. Chem. Phys. 98, 5648-5652 (1993).

101. C. Lee, W. Yang, R. G. Parr, Development of the Colle-Salvetti correlation-energy formula into a functional of the electron density. Phys. Rev. B. 37, 785-789 (1988).

102. P. J. Stephens, F. J. Devlin, C. F. Chabalowski, M. J. Frisch, Ab Initio calculation of vibrational absorption and circular dichroism spectra using density functional force fields. J. Phys. Chem. 98, 11623-11627 (1994).

103. S. Grimme, S. Ehrlich, L. Goerigk, Effect of the damping function in dispersion corrected density functional theory. J. Comput. Chem. 32, 1456-1465 (2011).

104. S. Grimme, J. Antony, S. Ehrlich, H. Krieg, A consistent and accurate ab initio parametrization of density functional dispersion correction (DFT-D) for the 94 elements HPu. J. Chem. Phys. 132 (2010), doi:10.1063/1.3382344.

105. F. Weigend, R. Ahlrichs, Balanced basis sets of split valence, triple zeta valence and quadruple zeta valence quality for $\mathrm{H}$ to Rn: Design and assessment of accuracy. Phys. Chem. Chem. Phys. 7, 3297-3305 (2005).

106. B. Mennucci, E. Cancès, J. Tomasi, Evaluation of solvent effects in isotropic and anisotropic dielectrics and in ionic solutions with a unified integral equation method: Theoretical bases, computational implementation, and numerical applications. J. Phys. Chem. B. 101, 10506-10517 (1997).

107. J. Tomasi, B. Mennucci, R. Cammi, Quantum mechanical continuum solvation models. Chem. Rev. 105, 2999-3093 (2005).

108. G. A. DiLabio, D. A. Pratt, Density Functional Theory Based Model Calculations for Accurate Bond Dissociation Enthalpies. 2. Studies of $\mathrm{X}-\mathrm{X}$ and $\mathrm{X}-\mathrm{Y}(\mathrm{X}, \mathrm{Y}=\mathrm{C}, \mathrm{N}, \mathrm{O}, \mathrm{S}$, Halogen) Bonds † . J. Phys. Chem. A. 104, 1938-1943 (2000). 